Reprod. Nutr. Dévelop., 1980, 20 (1 B), 323-329.

\title{
Effet à court terme de la prolactine sur son récepteur
}

\author{
par J. DJIANE, C. DELOUIS, P. A. KELLY* \\ avec la collaboration de Lucetfe BELAIR et Marie-Louise FONTAINE \\ Laboratoire de Physiologie de la Lactation, I.N.R.A. \\ 78350 jouy en Josas, France. \\ * M.R.C. Group in Molecular Endocrinology \\ C.H.U.L., Laval, Québec, GIV4G2, Canada.
}

Summary. Short-term effect of prolactin on its receptors.

It is known that prolactin (PRL) when injected chronically is able to increase the level of its own receptors. We have shown here that, after a single IV injection of PRL to lactating rabbits, total prolactin-receptor levels (measured after in vitro desaturation of injected prolactin by a $\mathrm{MgCl}_{2}$ treatment of the membranes) decreased from 41.6 to $18.4 \mathrm{p}$. 100 (specific binding) in $6 \mathrm{hrs}$. The same results were obtained in organ-culture of the rabbit mammary gland in the presence of prolactin ; in addition, the down-regulation of prolactin receptors could be counteracted by lysosomotropic agents (chloroquine $\mathrm{NH}_{4} \mathrm{Cl}$ ) in vitro.

These results showed that prolactin could, at short term, induce a down regulation of its receptors, and that this effect might be related to an endocytosis of hormone-receptor complex and lysosome degradation.

La prolactine possède des récepteurs dans de nombreux tissus (Posner et al., 1974a). La glande mammaire et le foie sont les organes réputés les plus riches en récepteurs à cette hormone. Dans la glande mammaire, le récepteur a été isolé et purifié (Shiu et Friesen, 1974). Les modulations hormonales du nombre de récepteurs ont été largement étudiées dans le foie (Posner et al., 1974b ; Costlow ef al., 1975) ef la glande mammaire (Djiane et al., 1977 ; Djiane et Durand, 1977) : Contrairement à la majorité des hormones étudiées (Insuline, GH, LH), pour lesquelles on a mis en évidence une régulation négative du nombre de récepteurs par leur hormone spécifique (" down regulation »), on a reconnu à la prolactine un effet stimulant de son propre récepteur soit dans le foie (Costlow et al., 1975), soit dans la glande mammaire (Djiane et Durand, 1977).

Récemment nous avons développé des techniques de désaturation des récepteurs prolactiniques soit in vivo (Djiane ef al., 1977), soit in vitro (Kelly ef al., 1979). La désaturation in vitro utilise les agents chaotropiques à forte molarité $\left(\mathrm{MgCl}_{2} 4 \mathrm{M}\right)$ ef permet la mesure du nombre total de récepteurs, les libres et ceux saturés par l'hormone endogène. 
Nous décrivons ici une étude utilisant la désaturation in vitro, réalisée en vue d'analyser l'effet à court terme de la prolactine sur le nombre de ses récepteurs. Le but de cette étude est de démontrer que, comme beaucoup d'autres hormones, la prolactine est capable d'induire une diminution de ses propres récepteurs et que cette diminution correspond à une dégradation lysosomiale du complexe hormone-récepteur.

\section{Matériel et méthodes.}

Animaux. - Nous avons utilisé des lapines de race Néo-Zélandaise soił à 7 10 jours de lactation (expériences in vivo), soit à 12 jours de pseudogestation (expériences en culture organotypique).

Préparation des membranes. - Le tissu mammaire est homogénéisé dans 3 volumes de sucrose $0,3 \mathrm{M}$, puis centrifugé à $12000 \mathrm{~g}$ (20 min). Le surnageant est recentrifugé à $100000 \mathrm{~g}$ (90 min), le culot de cette dernière centrifugation, qui contient la majorité des membranes de la cellule est repris dans un tampon tris $25 \mathrm{mM}, \mathrm{MgCl}_{2}, 10 \mathrm{mM}$, $\mathrm{pH} 7.5$ et conservé congelé jusqu'à utilisation.

Test de liaison spécifique. - La prolactine (PRL NIH PS12) ou l'hormone de croissance humaine (hGH NIH) qui est lactogène chez la lapine et utilise le même récepteur que la prolactine, sont iodées à lode 125 à l'aide de chloramine T. $100000 \mathrm{cpm}$ de ${ }^{125} \mathrm{I}$ PRL ou de ${ }^{125} \mathrm{~h} \mathrm{hGH}$ sont incubés en présence ou en absence de $1 \mu \mathrm{g}$ de PRL non marquée pendant $16 \mathrm{~h}$ avec $200 \mu \mathrm{g}$ de protéines membranaires. Après incubation, les membranes et le milieu d'incubation sont séparés par centrifugation et la radioactivité du culot membranaire est comptée en rayonnement $\gamma$; les résultats sont exprimés en pourcentage de liaison spécifique par rapport à la quantité totale de radioactivité présente. La détermination des récepteurs totaux est réalisée après désaturation in vitro : les membranes sont préalablement incubées avec une solution de chlorure de magnésium (4 M) afin de dissocier la prolactine de ses récepteurs; les membranes ont ensuite lavées puis incubées avec la prolactine radioactive comme précédemment.

\section{Résultats.}

\section{Expérimentation in vivo.}

La figure $1 a$ montre qu'après injection intraveineuse d'une dose unique de prolactine (100 UI) à des lapines en lactation, les récepteurs à la prolactine sont rapidement occupés. L'occupation maximale est observée à $15 \mathrm{~min}$ et les récepteurs reviennent à leur niveau initial $30 \mathrm{~h}$ après l'injection.

Les concentrations circulantes de prolactine sont très importantes : un pic est observé $1 \mathrm{~min}$ après injection, suivi d'une décroissance très rapide. Bien que la prolactine circule à des concentrations saturantes 15 min après injection, 20 p. 100 des récepteurs restent inoccupés. Ce qui correspond à une inaccessibilité à l'hormone d'une partie des récepteurs. Contrairement à la très lente et incomplète dissociation de la prolactine de son récepteur observée in vitro sur des membranes isolées, une apparente réversibilité est remarquée in vivo, puisque la dissociation débute entre 15 min 
et $1 \mathrm{~h}$. Cette apparente dissociation, in vivo, peut correspondre en réalité à de nouveaux récepteurs récemment synthétisés. La figure $1 b$ montre chez les mêmes animaux l'évolution des récepteurs totaux, c'est-à-dire mesurés après désaturation in vitro par traitement des membranes au $\mathrm{MgCl}_{2} 4 \mathrm{M}$. On observe une diminution progressive des récepteurs jusqu'à $6 \mathrm{~h}$ après injection. II existe un décalage entre l'évolution de l'occupation des récepteurs (fig. 1a) et l'évolution des récepteurs totaux (fig. 1b). En effet, alors que l'occupation maximale se produit à $15 \mathrm{~min}$, la diminution maximale des récepteurs n'est observée qu'à $6 \mathrm{~h}$.

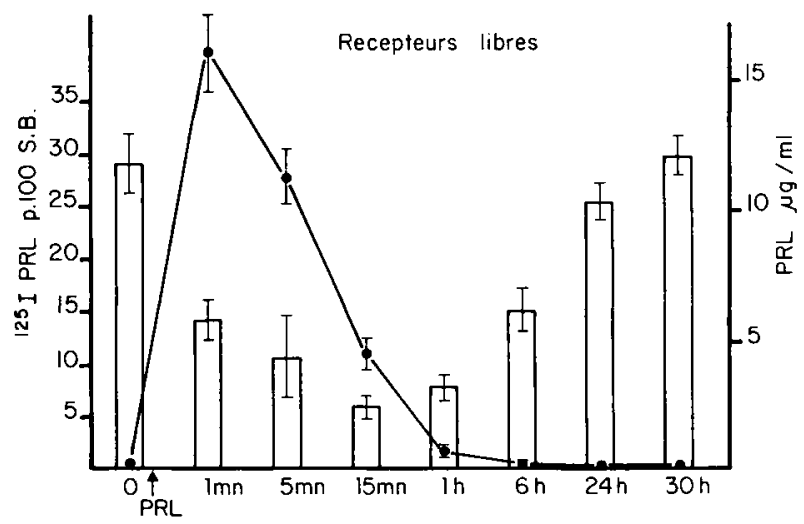

FIG. $1 a$.

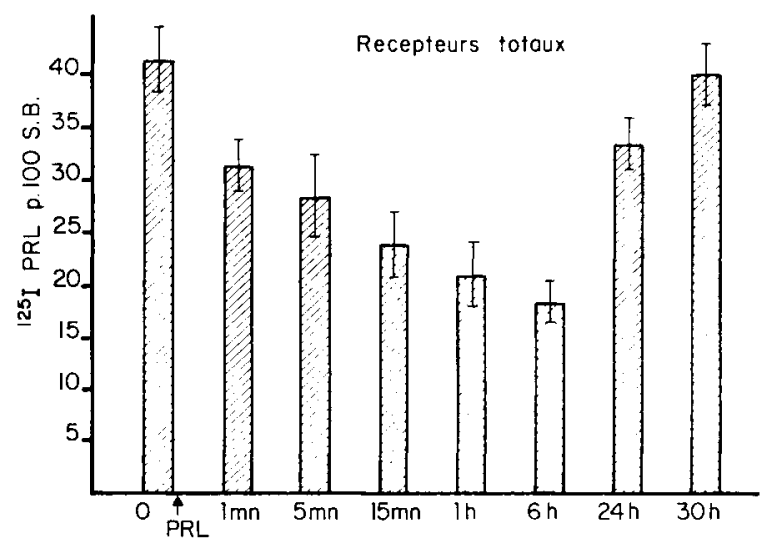

FIG. $1 b$.

FIG. 1. - Effet d'une injection intraveineuse de $3 \mathrm{mg}$ de prolactine sur le niveau de récepteurs à lo prolactine libres (fig. 1a) et totaux (fig. 1b). Des biopsies ( $\mathrm{g}$ environ) de glande mammaire sont réalisées sur des lapines en lactation aux différents temps indiqués. Les membranes sont préparées à partir de ces biopsies et $400 \mu \mathrm{g}$ de protéines sont incubées avec $100000 \mathrm{cpm}$ de ${ }^{125} \mathrm{~h} \mathrm{hGH}$ en absence ou en présence de $1 \mu \mathrm{g}$ de PRL, afin de déterminer le pourcentage de liaison spécifique. Une autre série de tubes est soumise à la désaturation in vitro $\left(\mathrm{MgCl}_{2} 4 \mathrm{M}\right)$ avant le test de liaison spécifique, représentant ainsi les niveaux des récepteurs totaux (fig. $1 b$ ). Les taux plasmatiques de prolactine suivant l'injection intraveineuse sont montrés sur la figure $1 a(\bullet-\bullet)$. 
La possibilité que la réduction du niveau des récepteurs totaux soit due à une réduction de l'efficacité du $\mathrm{MgCl}_{2}$ à dissocier la prolactine de ses récepteurs à leur maximum de saturation, est exclue du fait que les récepteurs libres augmentent entre $1 \mathrm{~h}$ et $6 \mathrm{~h}$, alors que les récepteurs tolaux continuent à diminuer.

\section{Expérimentation in vitro.}

a) Maintien du récepteur in vitro. - La figure 2 illustre le maintien du récepteur à la prolactine en culture organotypique de glande mammaire dans un milieu entièrement synthétique. Sans autre intervention hormonale que l'insuline $(5 \mu \mathrm{g} / \mathrm{ml})$, le récepteur se maintient pratiquement au niveau mesuré au moment de la mise en culture pendant plus de $48 \mathrm{~h}$. Ce maintien n'est pas un phénomène statique mais correspond à un équilibre entre la dégradation ef la synthèse de nouveaux récepteurs.

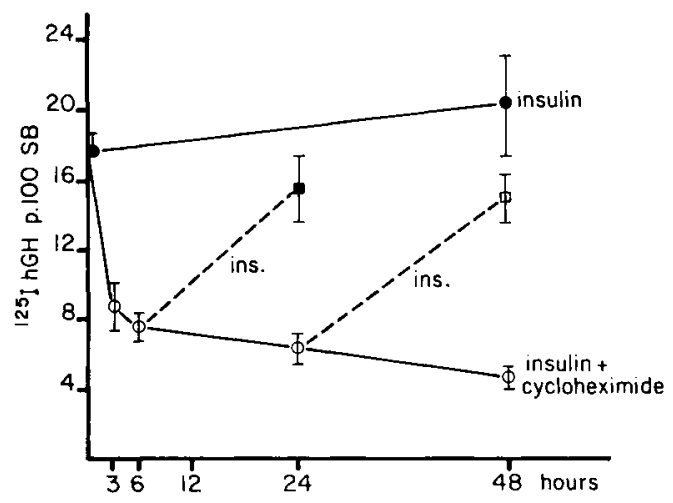

FIG. 2. - Effet de la cycloheximide $(1 \mu \mathrm{g} / \mathrm{ml})$ sur le maintien en culture organotypique du récepteur à la prolactine dans la glande mammaire de lapine explantée à 12 jours de pseudogestation.

En effet, si on ajoute un inhibiteur de la synthèse protéique (cycloheximide $1 \mu \mathrm{g} / \mathrm{ml}$ ) on observe très rapidement une diminution du niveau de récepteur (plus de 50 p. 100 en $3 \mathrm{~h}$ ), ce qui suggère un très rapide renouvellement du récepteur dans la glande mammaire. L'effet de la cycloheximide est réversible puisque l'on observe après son retrait une remontée du niveau de récepteurs en $24 \mathrm{~h}$.

FIG. 3. - Effet en culture organotypique de la prolactine sur le niveau de son récepteur dans la glande mammaire de lapine explantée à 12 jours de pseudogestation.

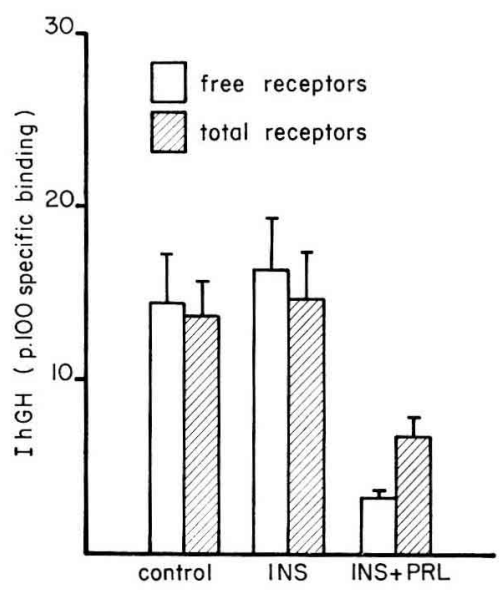


b) Effet de la prolactine. - La figure 3 montre le rôle en culture de glande mammaire de la prolactine sur le niveau de son propre récepteur et confirme les résultats obtenus in vivo. Après culture $(24 \mathrm{~h})$ en présence de prolactine $(1 \mu \mathrm{g} / \mathrm{ml}), 80 \mathrm{p} .100$ des récepteurs sont occupés. Les récepteurs totaux, titrés après désaturation in vitro, diminuent également de plus de 50 p. 100.

c) Effet des inhibiteurs des enzymes lysosomiales. - La chloroquine ou le chlorure d'ammonium sont reconnus capables d'inhiber les enzymes lysosomiales (De Duve ef al., 1974). La figure 4 montre qu'après $24 \mathrm{~h}$ de culture en présence de $1 \mu \mathrm{g}$ de prolactine, 50 p. 100 des récepteurs ont disparu, comme il a été précédemment décrit.

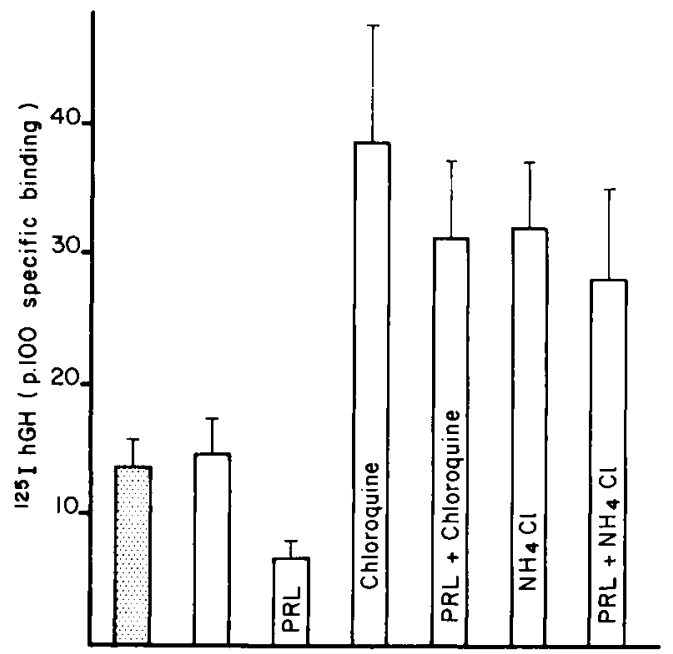

FIG. 4:- Effet des agenls lysosomotropes sur le niveau de récepteurs à la prolactine dans lo glande mammaire de lapine explantée à 12 jours de pseudogestation et cultivée pendant $24 \mathrm{~h}$. La chloroquine $10^{-4} \mathrm{M}$ ou le chiorure d'ammonium $10 \mathrm{mM}$ sont ajoutés au milieu de culture qui contient déjà soit de l'insuline $(5: \mathrm{g} / \mathrm{ml})$, soił de l'insuline plus de la prolactine $(1 \mathrm{~kg} / \mathrm{ml})$. Seuls les récepteurs totaux (mesurés après désaturation in vitro) sont figurés ici.

] = liaison spécifique avant culture $\square=$ liaison spécifique après culture $24 \mathrm{~h}$ en présence d'insuline.

Lorsque l'un ou l'autre des inhibiteurs des lysosomes est ajouté au milieu de culture, la dégradation des récepteurs est presque totalement stoppée, ce qui se traduit par une augmentation nette du nombre des récepteurs. Les inhibiteurs des enzymes lysosomiales, empêchant la dégradation du récepteur, sont capables d'inhiber la régulation négative observée avec la prolactine seule.

\section{Discussion.}

L'ensemble de ces résultats montre que la prolactine, comme beaucoup d'autres hormones étudiées, est capable d'induire, à court terme, une diminution de ses propres récepteurs dans la glande mammaire. La figure 5 schématise la double action de la pro- 
lactine sur la régulation de ses récepteurs, une augmentation à long terme par stimulation de la synthèse de nouveaux récepteurs (régulation positive) ef une diminution à court terme par stimulation de la dégradation du complexe hormone-récepteur (régulation négative). La stimulation par la prolactine de la synthèse de nouveaux récepteurs constitue l'hypothèse la plus vraisemblable pour le mécanisme de la régulation posifive, mais cette hypothèse n'a pas encore été vérifiée.

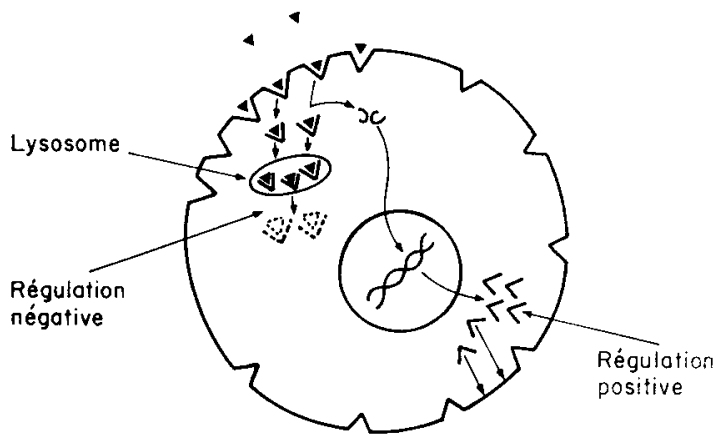

FIG. 5. - Représentotion schématique du double mécanisme de régulation des récepteurs ò la prolactine dans la glande mammaire.

Contrairement à ce que l'on observe pour la LH par exemple, la régulation négafive des récepteurs à la prolactine ne se produit que pendant une courte période ( 6 à 12 h) et avec une amplitude limitée. Elle ne réalise donc pas une réelle désensibilisation de l'organe effecteur. Cette rapide récupération est en accord avec le renouvellement rapide des récepteurs qui a été mis en évidence in vitro (fig. 2).

Quel est le mécanisme de cette régulation négative ? La prolactine a été localisée à l'intérieur de la cellule mammaire (Nolin et Witorsch, 1976). Récemment, des récepteurs à l'insuline et à la prolactine ont été identifiés dans des fractions golgiennes de foie de rat (Bergeron ef al., 1978). Pour la LH dans l'ovaire ou le testicule, il est clairement établi que la liaison de l'hormone est suivie de l'internalisation du complexe hormone-récepteur (Conn et al., 1978), puis de la dégradation de ce complexe dans les lysosomes (Ascoli et Puett, 1978). Les expériences que nous avons rapportées, utilisant des agents stabilisant les lysosomes, suggèrent qu'il en est de même pour la prolactine dans la glande mammaire. Ainsi la régulation négative des récepteurs à la prolactine aurait pour mécanisme une compartimentation rapide du complexe hormonerécepteur de la membrane plasmique vers les lysosomes suivie de la dégradation de ce complexe. Le rôle de cette régulation négative dans le mécanisme d'action de la prolactine (pour laquelle on ne connaît pas de second messager intracellulaire) reste à déterminer.

5e Réunion du groupe Développement I.N.R.A., Clermont-Ferrand/Theix, 17-18 mai 1979.

\section{References}

ASCOLI M., PUETT D., 1978. Degradation of receptor-bound human choriogonadotropin by murine leydig tumor cells. J. biol. Chem., 253, 4892-4899.

BERGERON J. J. M., POSNER B. I., JOSEFSBEG Z., SIKSTROM J., 1978. Intracellular polypeptide hormone receptors. The demonstration of specific binding sites for insulin and human growth hormone in Golgi fractions isolated from the liver of female rats. J. biol. Chem., 253, 4058-4066. 
CONN P. M., CONTI M., HARWOOD J. P., DUFAU M. L., CATT K. J., 1978. Internalisation of gonadotrophin receptor complex in ovarian luteal cells. Nature, 274, 598-600.

COSTLOW M. F., BUSCHOW R. A., MCGUIRE W. L., 1975 . Prolactin stimulation of prolactin receptors in rat liver. Life Sci., 17, 1457-1466.

DE DUVE C., DE BARSY T., POOLE B., TROUET A., TULKENS P., VAN HOOF, 1974. Lysosomotropic agents. Biochem. Pharmacol., 23, 2495-2531.

DJIANE J., DURAND P., 1977. Prolactin progesterone antagonism in self regulation of prolactin receptors in the mammary gland. Nature, 266, 641-643.

DJIANE J., DURAND P., KELLY P. A., 1977. Evolution of prolactin receptors in rabbit mammary gland during pregnancy and lactation. Endocrinology, 100, 1348-1356.

KELLY P. A., LEBLANC G., DJIANE J., 1979. Estimation of total prolactin binding sites after in vitro desaturation. Endocrinology. 104, 1631-1638.

NOLIN J., WITORSCH R. J., 1976. Detection of endogenous immunoreactive prolactin in rat mammary epithelial cells during lactation. Endocrinology, 99, 949-958.

POSNER B. I., KELLY P. A., SHIU R. P., FRIESEN H. G., 1974a. Studies of insulin, growth hormone and prolactin binding : tissue distribution, species variations and characterization. Endocrinology, 96, 521-531.

POSNER B. I., KELLY P. A., FRIESEN H. G., 1974b. Induction of a lactogenic receptor in rat liver. Influence of oestrogen and the pituitary. Proc. nat. Acad. Sci. USA, 71, 2407-2410.

SHIU R. P. C., FRIESEN H. G., 1974. Solubilization and purification of a prolactin receptor from. rabbit mammary gland. J. biol. Chem., 249, 7902-7910. 